\title{
The scientific basis for regarding vitamin $A$ and its analogues as anti-carcinogenic agents
}

\section{By R. Marian Hicks, School of Pathology, Middlesex Hospital Medical School, London $W_{\mathrm{I}} P{ }_{7} L D$}

Vitamin A (retinol) is an essential nutrient. Fortunately, it occurs in numerous food sources, both animal and vegetable, either as the pre-formed vitamin, retinol, or as $\beta$-carotene which can be converted to retinol. In the UK, where most people have an adequate diet, the effect of acute deficiency is seldom seen. However, analyses of diets show a substantial percentage of the population with multiple vitamin deficiencies, including deficiency of vitamin $A$, particularly in the older age groups (Department of Health and Social Security, 1979). These mild deficiencies are unlikely to produce any overt symptoms of disease. However, epidemiological evidence shows there to be a tendency for some human-cancer risks to be inversely associated with blood retinol; people with above-average blood retinol levels, or above-average $\beta$-carotene intakes, are at low average risk of cancer (Bjelke, I 975; Wald et al. 1980; Peto et al. 1981).

It has been known for more than 50 years that vitamin $A$ is essential to maintain the normal differentiation of specialized epithelial tissues throughout the body (Wolbach \& Howe, 1925). Deficiency of vitamin A results in failure of differentiation and therefore failure of normal function, and in many epithelia the tissue becomes keratinized. For example, the epithelia lining the trachea, bronchus, vagina, mouth and bladder are replaced with an epithelium which appears more like normal skin (Hicks, 1968, 1969). It is worth noting that although vitamin A deficiency prevents normal differentiation of epithelial tissues, it does not inhibit cell proliferation and, indeed, vitamin A deficiency is usually accompanied by hyperplasia of the affected tissue.

The keratinization of vitamin A deficient epithelia is reversible once the normal levels of vitamin $A$ are restored and this is true both in vivo and in vitro in organ culture. Thus, addition of retinyl acetate to the incubation of media of prostatic cancer cultured in vitro, reversed the squamous metaplasia and produced normally differentiated tubules (Lasnitzki, 1955). Since carcinomas, i.e. malignancies of epithelial tissues as opposed to malignancies of supporting tissues or leukaemias, represent $95 \%$ of fatal malignancies in man, this was a particularly interesting observation. Development of carcinomas always involves alterations in the normal differentiation of the tissue. Indeed, cancer is regarded by some people as a disease of differentiation. It thus seemed possible that vitamin $A$, or its analogues, known collectively as retinoids (Sporn, Dunlop, Newton \& Smith, 1976) could be anticarcinogenic by reinforcing expression of the normal phenotype and thus, at the same time, preventing expression of the new malignant phenotype. Implicit in this suggestion is the possibility that retinoids function like steroid hormones, to 
control phenotypic expressions. Unlike promoters, which are thought to increase the likelihood of random expression of the genome, they would act by reinforcing the normal expression. The idea thus arose that vitamin $A$ might be used as a tumour-preventive agent for human cancer. Unfortunately, however, naturallyoccurring retinol and retinoic acid are toxic at high doses and, in the light of current knowledge, should not be employed therapeutically for the chemoprevention of cancer in man.

\section{Retinoid toxicity}

Retinol is accumulated by the liver, and prolonged, chronic ingestion of highdoses of vitamin $A$ results in the destruction of liver parenchymal cells. Ingestion of acute high doses may cause brain-stem oedema, leading to headaches then coma followed by death. The major consequences of excessive vitamin $A$ intake have been described in several reviews (Bollag, 1975; Mandel, 1975). Acute vitamin A poisoning is now rare but as recently as 1912 , vitamin A poisoning claimed the life of Dr Xavier Mertz on the expedition led by Dr Douglas Mawson to the Australasian Antarctic. On the overland trip Mawson and Mertz were reduced to near-starvation and were forced to kill and eat their sleigh dogs which were tough to the point of being almost indigestible. However, they were able to chew and swallow the livers, even though they were unpalatable, and thereafter they became very ill indeed. Their skin peeled from head to foot and even the thick skin on the soles of their feet completely desquamated to leave their feet raw and bleeding. Mertz died, but Mawson survived after losing his hair and being reduced to a terrible state, both psychologically and physically. Interestingly, however, after being rescued and properly fed he survived and his health was completely restored. Such consequences of high vitamin $A$ intake preclude the possibility of considering it seriously as a therapeutic or preventive agent for cancer therapy.

In an attempt to overcome the toxicity problem, more than 1000 retinoid analogues have been synthesized which, by virtue of alteration to their chemistry, yield biologically active analogues (Sporn \& Newton, I98I). Many of these have been assayed for toxicity, e.g. by the tracheal organ culture assay (Clamon et al. I974; Sporn, Dunlop, Newton \& Henderson, 1976), and some of the most promising have been tested in vivo as anti-carcinogenic agents in experimental animal systems. Unfortunately, in vivo none of the retinoids are completely nontoxic when used at high-dose levels. Characteristic symptoms of retinoid toxicity include reduced weight gain, thinning and increased fragility of the long bones, and alterations in haematological properties (Hixson \& Denine, I 978a). However, some of the newer analogues can be well tolerated by experimental animals when used at relatively low-dose levels.

\section{The anti-carcinogenic potential of retinoids in experimental animals}

In order to assess the anti-carcinogenic potential of retinoids, they have been given to carcinogen-treated experimental animals. On the basis of tumour incidence, usually after a relatively short experimental time, some of these retinoids have been shown to 'prevent' or 'inhibit' the development of cancer in the rodent 
bladder, breast and skin in response to proven carcinogens (Sporn \& Newton, I 979). In particular, Sporn and Moon and their colleagues found that in the shortterm, the development of bladder cancer in rats and mice could be delayed or even halted by retinoids (Grubbs et al. 1977; Sporn et al. 1977; Squire et al. 1977; Becci, Thompson, Grubbs, Brown et al. 1979; Becci, Thompson, Grubbs, Squire et al. 1979; Becci et al. 1981 ; Thompson et al. 1981). Most of these experiments involved pre-treating the animals with high doses of either $N$-methyl- $N$ nitrosourea (MNU) or with $N$-butyl- $N$-(4-hydroxybutyl)nitrosamine (BBN) and assessment of the carcinogenic response at a single time, some months after the animals had been maintained on a retinoid-containing diet. Thus, Grubbs et al. (1977), Becci, Thompson, Grubbs, Squire et al. (1979), Becci et al. (198I) and Thompson et al. (198I) terminated their experiments after approximately $4 \frac{1}{2}$ months on the retinoid diet; Sporn et al. (1977), Squire et al. (1977) and Murasaki et al. (1980) after 8 months; Tannenbaum et al. (1979) after 9 months and Becci, Thompson, Grubbs, Brown et al. (1979) after 9-I I months. In all these experiments, the inhibition of bladder carcinogenesis was not complete but there was a consistent reduction in incidence of papillomas and carcinomas found at autopsy and the overall differentiation of the tumours and the rest of the urothelium was better in the retinoid-fed than in the placebo-fed groups. However, because most of these experiments were relatively short-term studies, it was not clear whether the animals would have been able to tolerate life-time dosing with retinoids, or whether the observed retinoid inhibition of carcinogenesis was transient, permanent, or persisted only as long as the retinoid was administered. Such information is essential in order to assess the potential therapeutic value of any particular retinoid as an anti-cancer agent. In the Middlesex Hospital Medical School we have undertaken a number of long-term feeding studies with retinoids to assess the response of both rats and mice to treatment with the specific bladder carcinogen, BBN.

\section{The long-term modulation by retinoids of the carcinogenic response of rodents to the specific bladder carcinogen, $B B N$}

In man, bladder cancer takes two different forms. The first and most common is papillary carcinoma, which characteristically is an exophytic, well-differentiated, slow-growing multi-focal cancer of the transitional epithelium (urothelium) which only eventually becomes locally invasive. The second and more life-threatening form is flat carcinoma in situ which frequently progresses to flat invasive carcinoma which is rapidly growing, malignant, metastatic and rapidly fatal. In general, the slow-growing papillary disease can be controlled for many years by local transurethral resection, but carcinoma in situ and flat invasive disease can only be treated by radium implants and/or total cystectomy and even then is frequently fatal because of widely disseminated metastases. Most human carcinomas of the bladder start as papillary growths, but as the disease progresses, it is not uncommon to find both types of tumour in the same bladder (Koss, 1979; Newman \& Hicks, 198 r). 
In rodents, there are both species- and strain-related differences in the response of the urinary bladder to carcinogens. Intragastric administration of BBN to Fischer 344 rats induces transitional cell carcinomas which are an excellent model of the slow-growing, well-differentiated papillary disease in man (Becci, Thompson, Grubbs \& Moon, 1979). By contrast, the hybrid $\mathrm{B}_{6} \mathrm{D}_{2} \mathrm{~F}_{1}$ mouse responds to the same carcinogen by production of carinomas in situ and highly invasive, poorly differentiated transitional cell carcinomas of the bladder which are an excellent model for the second form of the disease seen in man (Becci $e t$ al. I 98 I).

Effect of 13 -cis-retinoic acid and $\mathrm{N}$-ethyl-retinamide on the response of the bladder in the Fischer 344 rat to treatment with BBN. Between 1977 and 1980, using the Fischer $344 \mathrm{rat} / \mathrm{BBN}$ experimental model for papillary transitional cell carcinoma of the bladder, we investigated the ability of two synthetic retinoids, namely I 3 -cis-retinoic acid and $\mathrm{N}$-ethyl-retinamide, to modulate carcinogenesis. The experimental details and results have been published elsewhere (Hicks $e t$ al. Ig82).

Both retinoids were well tolerated and did not reduce the life-span of the animals over a period of 2 years but they did have some mild toxic effects, in particular on the skeleton. Both compounds caused thinning of the long bones but there were no spontaneous fractures. There was also a decrease in the weight gain of the animals. In this experimental bladder cancer model the carcinogenic response in terms of the total tumour volume was directly proportional to the dose of carcinogen used. The latent period between administration of carcinogen and the start of exponential tumour growth was inversely proportional to the dose of carcinogen. The results of our experiments demonstrated that both retinoids lengthened the latent period before exponential tumour growth started by approximately 8 to ro weeks. They appeared to hold the tissue in the latent period so that, at any point in time, the tumours in the retinoid-fed animals were 'younger' than those in the placebo-fed animals and consequently were both smaller and better differentiated. When the animals were permitted to survive a second year, it was found that retinoids did not prevent cancer development, nor prevent squamous metaplasia or markers of neoplasia from developing. 'They did not reduce the rate of tumour growth once the cancers had started to grow and they did not prevent the animals from dying of bladder cancer. They did, however, lengthen the period of symptomfree urothelial hyperplasia before tumour growth commenced and thus reduced the age-related incidence of bladder cancer. This is potentially very useful and could equate to approximately 5 years of symptom-free life in man. On the basis of such experiments these retinoids could well be used as an additional modality for treating patients at risk for bladder cancer and could be particularly useful in the group of people who have already had one bladder cancer and thus are at high risk of developing neoplasms at other sites in the bladder.

The effect of tetrazol-5-yl retinamide and N-4-hydroxyphenyl-retinamide on the carcinogenic response of the $B 6 D_{2} F_{1}$ mouse to the bladder carcinogen, $B B N$. Using the $\mathrm{B} 6 \mathrm{D}_{2} \mathrm{~F}_{1}$ mouse/ $\mathrm{BBN}$ model for flat invasive carcinoma of the bladder, we are 
in the process of investigating the potential anti-carcinogenic effect of two relatively new retinoids, namely, tetrazol-5-yl retinamide and $\mathrm{N}-4$-hydroxyphenylretinamide. The latter compound has already been demonstrated by Moon and his colleagues (1979) to be effective in preventing the development of breast cancer in an experimental rat mammary gland model. In that system it also had a very significant anti-proliferative effect and prevented the development of the normal ductular epithelium.

Only preliminary results are available so far. However, they show that these retinoids also cause some reduction in weight gain and also thinning of the long bones but do not apparently increase mortality. These retinoids were given to the mice at a concentration of approximately $\mathrm{I} \mathrm{mm}$ in the diet, the same concentration as that given to rats in the previous experiments. Mice, however, eat more in proportion to their body-weight than do rats, so whereas the rats were eating approximately $15 \mathrm{mg}$ retinoid/ $\mathrm{kg}$ body-weight $/ \mathrm{d}$, the mice ate $60 \mathrm{mg} / \mathrm{kg}$ bodyweight/d, a four-fold increase. The relatively mild toxic effects observed in mice with the 4-hydroxyphenyl-retinamide and tetrazol-5-yl retinamide indicate that the mouse may be less sensitive than the rat to retinoid toxicity and/or these two retinoids are per se less toxic than 13 -cis-retinoic acid and $N$-ethyl-retinamide.

Based on results obtained so far, after only 5 months' feeding of these two retinoids to mice, there is a clear difference in the response to the carcinogen in the placebo-fed and retinoid-fed animals and, furthermore, a marked difference in activity of the two compounds (Tables $I$ and 2 ).

Nineteen weeks after completing the carcinogen treatment, the placebo-fed animals had a high incidence $(66 \%)$ of transitional cell carcinoma of the bladder, and a further $22 \%$ had a hyperplastic or dysplastic urothelium. This ratio was not significantly altered by feeding tetrazol-5-yl retinamide in the diet $(62 \%$ and $20 \%$ respectively), but the 4 -hydroxyphenyl-retinamide reversed the ratio and effectively reduced the age-related cancer incidence, so that only $25 \%$ had carcinoma of the urothelium and $49 \%$ had hyperplasia (Table $\mathrm{I}$ ). When the carcinogenic response

\section{Table 1. Effect of two retinoids on the incidence of hyperplasias and bladder cancers induced in female $B 6 D_{2} F_{1}$ mice by $30 \mathrm{mg} \mathrm{BBN}$}

(Numbers of animals with hyperplasia or cancer/total number of animals in group shown in parentheses)

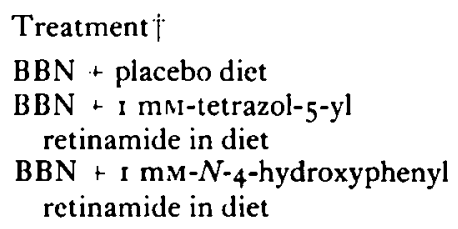

$\begin{array}{cc}\begin{array}{c}\text { Percentage incidence of } \\ \text { urothelial hyperplasia }\end{array} & \begin{array}{c}\text { Percentage incidence of } \\ \text { bladder cancer }\end{array} \\ 22(10 / 47) & 66(3 \mathrm{r} / 47) \\ 20(9 / 45) & 62(28 / 45) \\ 49(25 / 51) & 25(13 / 51)\end{array}$

-BBN, $N$-butyl- $N$-(4 4 -hydroxybutyl)nitrosamine.

The carcinogen was given by mouth as 10 weekly portions of $3 \mathrm{mg}$ each. The animals were then given a diet containing $\mathrm{I} \mathrm{m} \mathrm{m}$ of the retinoid, or a placebo diet, for 19 weeks. 
Table 2. Effect of two retinoids on the stage of bladder cancer induced in female $B 6 D_{2} F_{1}$ mice by $30 \mathrm{mg} B B N^{*}$

(Number of animals with carcinoma/total number of animals in group shown in parentheses)

\begin{tabular}{|c|c|c|c|}
\hline 'I'reatment $\uparrow$ & $\begin{array}{l}\text { Percentage } \\
\text { incidence of } \\
\text { carcinoma in situ }\end{array}$ & $\begin{array}{c}\text { Percentage } \\
\text { incidence of } \\
P_{1} \text { and } P_{2} \text { carcinomas }\end{array}$ & $\begin{array}{c}\text { Percentage } \\
\text { incidence of } \\
\mathrm{P}_{3} \text { carcinomas }\end{array}$ \\
\hline BBN + placebo diet & $28(13 / 47)$ & $32(15 / 47)$ & $6(3 / 47)$ \\
\hline $\begin{array}{l}\mathrm{BBN}+\text { I mis-tetrazol-5-yl } \\
\text { retinamide in diet } \\
\text { BBN + I mM-N-4-hydroxyphenyl }\end{array}$ & $3^{8}(17 / 45)$ & $13(6 / 45)$ & I I $(5 / 45)$ \\
\hline retinamide in diet & $2(I / 5 I)$ & $12(6 / 51)$ & I $2(6 / 5 \mathrm{I})$ \\
\hline
\end{tabular}

*BBN, $N$-butyl- $N$-(4-hydroxybutyl)nitrosamine.

$\uparrow$ The carcinogen was given by mouth as 10 weekly portions of $3 \mathrm{mg}$ each. The animals were then fed a diet containing I $\mathrm{m} M$ of the retinoid, or a placebo diet, for I 9 weeks.

is analysed in terms of the stage of the tumour (i.e. the depth of penetration of the neoplastic urothelium into the bladder wall, classified according to the World Health Organization system (see Pugh, 1973)) differences in the mode of action of the two retinoids are indicated (Table 2). The characteristic failure of normal differentiation following carcinogen treatment is illustrated by the very high percentage incidence of carcinoma in situ in the placebo-fed animals $(28 \%)$. The incidence of carcinoma in situ was actually increased by the tetrazol-5-yl retinamide to $38 \%$, but the 4 -hydroxyphenyl-retinamide produced a significantly better differentiated urothelium. Indeed, only a single animal was found to have carcinoma in situ in the group given this retinoid. The tetrazol-5-yl retinamide, however, was not entirely without effect on the neoplastically transformed urothelium and in the group given this compound, fewer cancers which had invaded the bladder wall ( $P_{1}$ and $P_{2}$ carcinomas) were found by comparison with the placebo-fed group, namely $13 \%$ as compared to $32 \%$. The percentage incidence of aggressive, highly invasive tumours which had passed right through the bladder wall and were spreading over its peritoneal surface $\left(\mathrm{P}_{3}\right.$ carcinomas) was not significantly affected by either compound (Table 2 ).

The numbers of persistent hyperplasias which develop in the mouse bladder in response to a carcinogen has been shown to be a prediction of the number of carcinomas which will develop several months later (Littlefield et al. 1979). The results obtained with 4-hydroxyphenyl-retinamide suggest it may be acting in two different ways on the urothelium. First, it has a dramatic effect on differentiation of the urothelium and maintains normal transitional celi differentiation, even in urothelia made hyperplastic by the carcinogen treatment. Second, and by analogy with the results of our previous rat-bladder experiments, the compound appears to be particularly effective in terms of prolonging the latent period of quiescent hyperplasia before rapid tumour growth commences. It must be emphasized that these results are preliminary and the experiments are not yet complete, and we do not yet know how long this retinoid can restrain the hyperplasias from developing 
into carcinomas. By contrast, the tetrazol-5-yl retinamide failed to maintain normal differentiation and the majority of the BBN-treated animals had dysplastic or neoplastic urothelia. However, this compound also clearly and significantly delays the time at which invasion of the bladder wall by the carcinoma commences, i.e. it retards the progression of the disease. Neither compound has any apparent effect on the incidence of rapidly growing, highly invasive, $\mathrm{P}_{3}$ carcinomas.

\section{Conclusions from these rodent experiments}

If the effects of retinoids on the response of the rat and mouse to the bladder carcinogen BBN are considered together, a number of interesting points can be concluded. Retinoids appear to be effective both against slow growing exophytic tumours, as in the rat model, and against flat invasive carcinomas, as in the mouse model. The experiments highlight the possibility of organ specificity of retinoids. The 4 -hydroxyphenyl compound was clearly far more effective in the bladder than the tetrazol-5-yl retinamide. However, the 4-hydroxyphenyl retinamide is also the most effective compound found so far as an anti-carcinogen in mammary carcinogenesis (Moon et al. 1979) so it is possible that this particular compound is actually more efficient per se than the others as an anti-carcinogen, irrespective of the organ. In the rat model we know that the retinoids we studied acted by prolonging the latent period during which there is persistent but quiescent hyperplasia of the urothelium. Once the restraint fails and the urothelium begins to grow, the retinoids have no effect on the rate of growth of the developing tumour. In the mouse model undoubtedly the 4 -hydroxyphenyl retinamide effectively delayed the development of cancer, but the fact that there was so much hyperplasia suggests that cancer will develop in these animals also, but after a longer period of time. This suggests, therefore, that the retinoids work in the mouse in the same way as in the rat, namely by delaying tumour growth by extending the latent period.

In vitro retinoids have been shown to antagonize the proliferative, tumourpromoting effects of phorbol esters in multi-stage skin carcinogenesis models. Specifically, they can inhibit induction of ornithine decarboxylase (EC 4.I.1.1 7) and of increased polyamine synthesis in response to phorbol ester promotion, but not in response to treatment with complete carcinogens (Boutwell \& Verma, 1979; Verma et al. 1980). It has become customary to regard retinoids as 'antipromoting' agents. However, both in the in vivo rat and mouse experiments described here, and in most other in vivo experiments published, the retinoids were not really tested as anti-promoting agents, since fully carcinogenic doses of the initiating carcinogen were used before the animals were put on the retinoidcontaining diet. In these experiments, the retinoids were not given at a time when they could act as anti-promoters, but were being investigated for their ability to delay tumour progression. This is a comparable situation to that in which they are likely to be used as therapeutic agents in man. All the positive anti-carcinogenic results obtained with retinoids in rodent models are explicable in terms of an increased latent period before tumour growth commences its exponential growth 
phase, rather than in terms of anti-promotion. Since the length of the latent period is inversely proportional to the dose of carcinogen used, if retinoids increase the length of this latent period the actual numerical results obtained will depend entirely on the time at which the animals are killed, with reference to the doseresponse curve for each dose of carcinogen (Hicks et al. 1982).

\section{The mechanism of the anti-carcinogenic action of retinoids}

There are various theories which can be considered to explain how retinoids extend the latent period in cancer development, but as yet we have no definite knowledge of the mechanism of action. One problem is to accommodate results such as those we have obtained with the mouse model, in which the retinoids may be effective in delaying the appearance of superficial $P_{1}$ tumours and their progression to locally invasive $P_{2}$ cancers, but do not alter the time-related incidence of the rapidly growing invasive $\mathrm{P}_{3}$ cancers. If one assumes a monoclonal origin for each cancer, this could reflect the severity of damage by the carcinogen to the genome in the neoplastically transformed stem cell. If the initial covalent reaction between the carcinogen and DNA is in a part of the genome which is not normally expressed (and statistically, this is the most likely situation), then a multistage carcinogenesis process of promotion and propagation will be required, in which several separate events are involved at the cellular level before the genetic damage can be expressed as a neoplastic phenotype (Hicks, 1980). If, however, the initial covalent reaction between the carcinogen and DNA is in an area of the genome in which immediate expression is possible (and this may be expected in a minority of cases), this could theoretically permit direct cancer development without the necessity of progressing through the various stages of promotion and propagation. If retinoids antagonize one or more of these steps in a multi-stage process, this may account for their ability to delay the appearance of some $P_{1}$ and $\mathrm{P}_{2}$ tumours. But if, as seems to be the case from our results, they have little or no effect on the fully committed cancer cell, this would support the suggestion that the aggressive $\mathrm{P}_{3}$ tumours in our system developed directly and did not progress through a multi-stage development process. Evidence is now beginning to accumulate that in a multi-stage system, development of neoplastic potential is associated with the production of growth regulatory polypeptides termed transforming growth factors (Sporn et al. $\mathrm{r} 98 \mathrm{I}$ ) which in vitro have been shown to confer neoplastic growth characteristics on non-neoplastic cells. It is thus very significant that retinoids have been reported to block phenotypic cell transformation by one of these factors, namely sarcoma growth factor (Todaro et al. 1978 ).

Evidence is also accumulating which indicates that immuno-potentiation by retinoids may be involved in their anti-carcinogenic action. Retinol and retinoic acid accelerate the rejection of allogeneic skin grafts (Floersheim \& Bollag, 1972; Jurin \& 'Tannock, r 972). Retinoic acid and other retinoids stimulate T-killer-cell induction in vitro (Dennert \& Lotan, 1978), increase T-cell cytotoxicity in vivo (Lotan \& Dennert, 1979), and stimulate mouse killer T-cells in allogeneic and 
syngeneic systems (Dennert $e t$ al. 1979). Clinically, retinoids have been claimed to have an immune-potentiating effect in the treatment of lung cancer (Mickshe et al. 1977). Since vitamin $A$ acts as an adjuvant in the immune response (Dresser, 1968 ) there is a basis for thinking that at least part of the anti-carcinogenic effect of retinoids may be attributable to an improvement in the hosts' immune-defence mechanisms.

There have been a few disturbing reports of enhancement, rather than inhibition of tumour growth by some retinoids, particularly by the naturally-occurring analogues, retinyl acetate and retinyl palmitate. Many of these can be attributed to the direct cytotoxic effect of topical application of high doses of retinoids to a tissue; following cytotoxic damage there is regenerative hyperplasia, which can act as a proliferative stimulus to any surviving, committed cancer cells. Levine \& Ohuchi ( 1978 ) have demonstrated that some, but not all, retinoids will induce an elevated prostaglandin synthesis in dog kidney cells in vitro. If high levels of retinoids will induce prostaglandin synthesis in vivo also, this could well account for some of their toxic effects. In support of this theory it has been shown that aspirin and a number of other non-steroidal, anti-inflammatory agents, which are known to inhibit prostaglandin synthesis, will protect mice against the toxic effect of retinoic acid (Harrison et al. 1977; Hixson \& Denine, 1978b). It is interesting, in this context, that the compound, 4 -hydroxyphenyl-retinamide, which we find to be the most effective anti-carcinogen in the mouse bladder cancer model, is a potent inhibitor of prostaglandin synthesis in the in vitro dog-kidney system.

\section{Conclusions}

In conclusion, the epidemiological data suggesting that a normal to high level of vitamin $A$ or $\beta$-carotene in the body may protect against cancer development in man, is supported by quite extensive experimental data on synthetic retinoids. In addition to the anti-carcinogenic effect of some retinoids on the induction of bladder cancer in rodent models, the anti-carcinogenic effect of retinoids has also been reported by other research groups working with experimental breast and skin cancer models. Research is currently in progress in America on the effect of retinoids on in vivo models for cancer of the pancreas, prostate, oesophagus and colon. The experimental evidence suggests that retinoids may well prove useful as chemo-preventive agents which, by shifting the time-related response to carcinogens, could perhaps achieve a useful reduction in age-related cancer incidence in man. They seem unlikely to be of therapeutic value in treating existing, growing cancers with the exception of skin cancer, where topical applications of high levels do have a direct therapeutic effect (Bollag \& Ott, I97I; Bollag, 1975).

The potential use of retinoids as chemo-preventive agents in the management of the cancer patient is a sufficiently attractive prospect to warrant further investigation of both natural and synthetic analogues and precursors of vitamin $A$. With a few exceptions, human cancers are ultimately uncontrollable by the currently available treatment modalities of surgery, radiotherapy and/or 
chemotherapy. In theory, it would appear feasible to use a retinoid to delay the progression of many histologically undetectable pre-neoplastic tumour foci into rapidly growing cancers, in combination with a cytotoxic drug which should kill the dividing neoplastic cells once they have escaped restraint. At the same time, it may be possible to limit the toxic side-effects of the retinoids with non-steroidal, anti-inflammatory agents such as aspirin or indomethacin. Experimental research using animal cancer models offers a challenge and potentially a very rewarding area for further research into the anti-carcinogenic potential of retinoids.

This project was funded with federal funds from the USA Department of Health and Human Services, under contract no. NOr $\mathrm{CP}_{75938}$. The contents of this publication do not necessarily reflect the views and policies of the Department of Health and Human Services. I am indebted to my colleagues who have worked with me on these problems over the past 5 years, namely J. Chowaniec, J. A. Turton, E. D. Massey, X. Tomlinson, J. Gwynne, A. Harvey, R. Wright and H. Ogbolu.

\section{REFERENCES}

Becci, P. J., 'I'hompson, C. J., Grubbs, C. J., Brown, C. C. \& Moon, R. C. (1979). Cancer Res. 39, 3141 .

Becci, P. J., Thompson, C. J., Grubbs, C. J. \& Moon, R. C. (1979). F. Natl. Cancer Inst. 62, I87. Becci, P. J., Thompson, C. J., Grubbs, C. J., Squire, R. A., Brown, C. C., Sporn, M. B. \& Moon, R. C. (1979). Cancer Res. 38, 4463 .

Becci, P. J., 'Thompson, H. J., Strum, J. M., Brown, C. C., Sporn, M. B. \& Moon, R. C. (1981). Cancer Res. 4I, 927.

Bjelke, E. (1975). Int. F. Cancer 15, 561 .

Bollag, W. (1975). Chemotherapy 21, 236.

Bollag, W. \& Ott, S. (1971). Cancer Chemother. Rep. 55, 59.

Boutwell, R. K. \& Verma, A. (1979). Pure Appl. Chem. 5 I, 857.

Clamon, G. H., Sporn, M. B., Smith, J. M. \& Saffiotti, U. (1974). Nature, Lond. 250, 64.

Dennert, G., Crowley, C., Kouba, J. \& Lotan, R. (1979). F. Natl. Cancer Inst. 62, 89.

Dennert, G. \& Lotan, R. (1978). Eur. F. Immunol. 8, 23.

Department of Health and Social Security (1979). Report on Health and Social Subjects. No. I6. Nutrition and Health in Old Age. London: HMSO.

Dresser, D. W. (1968). Nature, Lond. 2 17, 527.

Floersheim, G. L. \& Bollag, W. (1972). Transplantation 15,564 .

Grubbs, C. J., Moon, R. C., Squire, R. A., Sparrow, G. M., Stinson, S. E., Goodman, B. G., Brown, C. C. \& Sporn, M. B. (1977). Science 198, 743.

Harrison, S. I., Hixson, E. J., Burdeshaw, J. A. \& Denine, E. P. (1977). Nature, Lond. 269, 5 I I.

Hicks, R. M. (1968). F. Utrastruct. Res. 22, 206.

Hicks, R. M. (1969). Y. Anat. 104, 327.

Hicks, R. M. (1980). Br. med. Bull. 36, 39.

Hicks, R. M., Chowaniec, J., Turton, J. A., Massey, E. D. \& Harvey, A. (1982). In Molecular Interrelations of Nutrition and Cancer, p. 419 [M. S. Arnott, J. van Eys and Y.-M. Wang, editors]. New York: Raven Press.

Hixson, E. J. \& Denine, E. P. (1978a). Toxicol. Appl. Pharmacol. 44, 29.

Hixson, E. J. \& Denine, E. P. (1978b). Toxicol. Appl. Pharmacol. 45, 317.

Jurin, M. \& Tannock, I. F. (1972). Immunology 23, 283.

Koss, L. G. ( 1979). IIum. Pathol. ro, 533 .

Lasnitzki, I. (1955). Br. F. Cancer 9, 434 . 
Levine, I. \& Ohuchi, K. (1978). Nature, Lond. 276, 274.

Littlefield, N. A., Greenman, D. L., Farmer, J. H. \& Sheldon, W. G. (1979). F. environ. Pathol. Toxicol. 3, 35 .

Lotan, R. \& Dennert, G. (1979). Cancer Res. 39, 55.

Mandel, H. G. (1975). In The Pharmacological Basis of Therapeutics, 5th ed., p. 1570 [L. S. Goodman and A. Gilman, editors]. New York: Macmillan.

Micksche, M., Cerni, C., Kokron, O., Titscher, R. \& Wrba, H. (I977). Oncology, 34, 234.

Moon, R. C., Thompson, H. J., Becci, P. J., Grubbs, C. J., Gander, R. J., Newton, D. L., Smith, J. M., Phillips, S. L., Henderson, W. R., Mullen, L. T., Brown, C. C. \& Sporn, M. B. (1979). Cancer Res. 39, 1339.

Murasaki, G., Miyata, Y., Babaya, K., Arai, M., Fukushima, S. \& Ito, N. (1980). Gann 71, 333.

Newman, J. \& Hicks, R. M. (1981). Scanning Electron Microscopy, 3, I.

Peto, R., Doll, R., Buckley, J. D. \& Sporn, M. B. (1981). Nature, Lond. 290, 201.

Pugh, R. C. B. (1973). Cancer 32, I 267.

Sporn, M. B., Dunlop, N. M., Newton, D. H. \& Henderson, W. R. (1976). Nature, Lond. 263, 110.

Sporn, M. B., Dunlop, N. M., Newton, D. L. \& Smith, J. M. (1976). Fedn Proc. Fedn Am. Socs exp. Biol. 35, I 332 .

Sporn, M. B. \& Newton, D. I. (1979). Fedn Proc. Fedn Am. Socs exp. Biol. 38, 2528.

Sporn, M. B. \& Newton, D. L. (1981). In Inhibition of Tumor Induction and Development, p. 7 I [M. S. Zedeck and M. Lipkin, editors]. New York: Plenum Press.

Sporn, M. B., Newton, D. L., Roberts, A. B., De Larco, J. E. \& Todaro, G. J. (198I). In Molecular Actions and Targets for Cancer Chemotherapeutic Agents, p. 54I [A. C. Sartorelli, editor]. New York: Academic Press.

Sporn, M. B., Squire, R. A., Brown, C. C., Smith, J. M., Wenk, M. L. \& Springer, S. (1977). Science 195,487 .

Squire, R. A., Sporn, M. B., Brown, C. C., Smith, J. M., Wenk, M. L. \& Springer, S. (1977). Cancer Res. 37, 293 .

Tannenbaum, M., Tannenbaum, S., Richelo, B. N. \& Trown, P. W. (1979). Scanning Electron Microscopy 1,673 .

Thompson, H. J., Becci, P. J., Grubbs, C. J., Shealey, Y. F., Stanek, E. J., Brown, C. C., Sporn, M. B. \& Moon, R. C. (198I). Cancer Res. 41, 933 .

Todaro, G. J., De Larco, J. E. \& Sporn, M. B. (1978). Nature, Lond. 276, 272.

Verma, A. K., Conrad, E. A. \& Boutwell, R. K. (1980). Carcinogenesis $1,607$.

Wald, N., Idle, M., Boreham, J. \& Bailey, A. (1980). Lancet ii, 8 I 3 .

Wolbach, S. B. \& Howe, P. R. (1925). F. exp. Med. 42, 753 . 